\title{
ON BOUNDED APPROXIMATION PROPERTIES OF BANACH SPACES
}

\author{
EVE OJA \\ Faculty of Mathematics and Computer Science, Tartu University \\ J. Liivi 2, EE-50409 Tartu, Estonia \\ E-mail: eve.oja@ut.ee
}

\begin{abstract}
This survey features some recent developments concerning the bounded approximation property in Banach spaces. As a central theme, we discuss the weak bounded approximation property and the approximation property which is bounded for a Banach operator ideal. We also include an overview around the related long-standing open problem: Is the approximation property of a dual Banach space always metric?
\end{abstract}

1. Introduction. The most celebrated problem in the Scottish Book, Problem 153, was posed by S. Mazur on November 6, 1936, with an exceptional prize - a live goose (see, e.g., [29, p. 231]). Problem 153 concerned approximation of continuous functions of two variables and was as follows. Given a continuous function $f=f(s, t)$ defined on $[0,1] \times$ $[0,1]$ and a number $\varepsilon>0$; do there exist numbers $a_{1}, \ldots, a_{n} ; b_{1}, \ldots, b_{n} ; c_{1}, \ldots, c_{n}$, with the property that

$$
\left|f(s, t)-\sum_{k=1}^{n} a_{k} f\left(s, b_{k}\right) f\left(c_{k}, t\right)\right|<\varepsilon
$$

for all $s, t \in[0,1]$ ?

Mazur knew, according to [37, that the positive answer to Problem 153 would imply that all compact operators between arbitrary Banach spaces can be approximated, in the norm topology for operators, by finite-rank operators. The latter problem, known as the approximation problem, goes back at least to the Polish School in Lwów. It had been considered as one of the central open problems of functional analysis. Recall that the approximation problem was solved, in the negative, by P. Enflo [10] in 1972 (see [20] for a photo with Mazur handing a white goose in a basket to Enflo, in Warsaw, 1972).

2010 Mathematics Subject Classification: Primary 46B28; Secondary 46-02, 46B20, 47B10, 47L20. Key words and phrases: Banach spaces, bounded approximation properties, Banach operator ideals, tensor norms.

The paper is in final form and no version of it will be published elsewhere. 
2. Approximation properties. Let $X$ and $Y$ be Banach spaces. We denote by $\mathcal{L}(X, Y)$ the Banach space of all bounded linear operators from $X$ to $Y$ and by $\mathcal{F}(X, Y)$ its subspace of finite-rank operators. Let $I_{X}$ denote the identity operator on $X$.

Recall that $X$ has the approximation property (AP) if there exists a net $\left(S_{\alpha}\right) \subset$ $\mathcal{F}(X, X)$ such that $S_{\alpha} \rightarrow I_{X}$ uniformly on compact subsets of $X$. If $\left(S_{\alpha}\right)$ can be chosen with $\sup _{\alpha}\left\|S_{\alpha}\right\| \leq \lambda$ for some $\lambda \geq 1$, then $X$ is said to have the bounded approximation property (BAP), more precisely, $X$ is said to have the $\lambda$-bounded approximation property $(\lambda$-BAP). If $\lambda=1$, then $X$ has the metric approximation property (MAP). From the definitions, it is clear that the MAP implies the BAP, and the BAP implies the AP.

Every Banach space with a Schauder basis has the BAP, and every Banach space with a monotone basis has the MAP. Indeed, let $X$ have a Schauder basis $\left(e_{k}\right)$. Let $P_{n}$ be the natural projections associated with the basis, i.e. $P_{n}\left(\sum_{k=1}^{\infty} a_{k} e_{k}\right)=\sum_{k=1}^{n} a_{k} e_{k}$. Then $P_{n} \rightarrow I_{X}$ uniformly on the compact subsets of $X$, and $X$ has the $\lambda$-BAP with $\lambda=\sup \left\|P_{n}\right\|$.

The AP and the MAP were deeply studied by A. Grothendieck in his famous Memoir [16]. He found eight important criteria for the AP and five for the MAP (see [16. Chapter I, pp. 165 and 179]). In "Proposition" 37 in [ibid., pp. 170-171], he proved among others that the approximation problem is equivalent to Mazur's Problem 153, and that it is also equivalent to the fact that all Banach spaces have the AP. In fact, Grothendieck's "Proposition" 37 contains 19 conditions which are all equivalent to the approximation problem. Enflo [10], in his counterexample to the approximation problem, constructed a separable reflexive Banach space without the AP. By "Proposition" 37, there also exists a closed subspace of $c_{0}$ without the AP (see, e.g., 38, for an explicit construction). Grothendieck [16, Chapter I, p. 182], admitted that he did not know whether the failure of the MAP for a Banach space implies the same for a closed subspace of $c_{0}$.

Concerning the history of approximation properties, the BAP was essentially considered already in Banach's book [2, p. 237], but in a more general setting of the bounded compact AP (see Section 5 below). The notions of the AP and the MAP were introduced by Grothendieck in his Memoir [16] as "la condition d'approximation" [16, Chapter I, p. 167] and "la condition d'approximation métrique" [ibid., p. 178]. After mentioning that he does not know any example of a Banach space without the MAP ("Je ne connais pas d'exemple d'espace de Banach qui ne possède pas la propriété d'approximation métrique"), Grothendieck also occasionally introduced the BAP as "la variante affaiblie de la propriété d'approximation métrique" [ibid., p. 182].

Grothendieck remarked [ibid.] that there would exist a Banach space without the BAP, provided that there exist Banach spaces having the BAP, but failing the $\lambda$-BAP for arbitrarily large $\lambda$. This idea was made explicit by T. Figiel and W. B. Johnson [12] in 1973. They succeeded in constructing a sequence of Banach spaces $X_{n}, n=$ $1,2, \ldots$, with the BAP but failing the $n$-BAP, in particular, failing the MAP, and observed that the direct $\ell_{2}$-sum $\left(\sum_{n=1}^{\infty} X_{n}\right)_{2}$ has the AP but fails the BAP. These were the first counterexamples showing that the AP, BAP, and MAP are, in general, different notions.

Nowadays, the counterexamples are known even inside the space $c_{0}$. Let $X_{J S}$ be the closed subspace of $c_{0}$ constructed by W. B. Johnson and G. Schechtman in 1996 (see [18, 
Corollary JS]). As shown by G. Godefroy in 2001 (see [14, Theorem VI.3]), $X_{J S}$ fails the MAP but has the 8 -BAP. In fact, $X_{J S}$ has the 6 -BAP as shown very recently by I. Zolk [45. Very recently, T. Figiel, W. B. Johnson, and A. Pełczyński 13, Corollary 1.13] constructed a closed subspace of $c_{0}$ which fails the BAP but has the AP; their construction relies on the Johnson-Schechtman space $X_{J S}$ and a new concept: the BAP for pairs of Banach spaces.

It is not known whether the notions of the AP, BAP, and MAP are different for dual spaces. This is a famous open problem that goes back to Grothendieck's Memoir [16]. Sometimes it is called the "AP-implies-MAP" problem, and it is as follows.

Problem 2.1 (see, e.g., [3, Problem 3.8]). Does the AP of the dual space $X^{*}$ of a Banach space $X$ imply the $M A P$ of $X^{*}$ ?

A short overview around Problem 2.1 will be given in Section 3. Actually, there are two related subproblems, both open: it is not known whether the AP in dual spaces implies the BAP, neither is known whether the BAP in dual spaces implies the MAP. For instance, the space $B V\left(\mathbb{R}^{n}\right)$ of functions with bounded variation on $\mathbb{R}^{n}$ has the BAP (a recent result due to [1]). It is known that $B V\left(\mathbb{R}^{n}\right)$ is a dual Banach space (see [39]).

Problem 2.2 (see [13, Problem 6.8]). Does BV $\left(\mathbb{R}^{n}\right)$ has the MAP?

Trying to approach the AP-implies-MAP problem, some new variants of the BAP have emerged in the recent years. We shall survey two of them: "the weak BAP", which was introduced by A. Lima and E. Oja 24 in 2005 (see Sections 3 and 4), and "the AP which is bounded for a given Banach operator ideal", which was introduced by $\AA$. Lima, V. Lima, and Oja 21] in 2009 (see Section 5). For a third very recent variant of the BAP, "the BAP for a pair $(X, Y)$ ", where $X$ is a Banach space and $Y$ is its closed subspace, due to Figiel, Johnson, and Pełczyński, we refer to their fundamental paper [13.

We refer the reader to an excellent survey [3] by P. Casazza for a state-of-the-art on approximation properties, as it was 10 years ago. For related information, the reader should refer to the very interesting recent book [7, where J. Diestel, J. H. Fourie, and J. Swart revisit the famous Grothendieck's Résumé.

3. The AP is always somewhat metric. Let $X$ and $Y$ be Banach spaces. We denote by $\mathcal{K}(X, Y)$ and $\mathcal{W}(X, Y)$ the subspaces of $\mathcal{L}(X, Y)$ of compact operators and weakly compact operators.

Throughout the Memoir [16], Grothendieck was interested in a question whether the AP could be somewhat metric. He proved (see [16, Chapter I, p. 141]), that if the dual space $X^{*}$ of a Banach space $X$ has the AP, then the following metric condition holds.

(G) For every Banach space $Y$ and for every operator $T \in \mathcal{W}(Y, X)$, there exists a net $\left(T_{\alpha}\right) \subset \mathcal{F}(Y, X)$ such that $\sup _{\alpha}\left\|T_{\alpha}\right\| \leq\|T\|$ and $T_{\alpha} \rightarrow T$ in the strong operator topology.

At the very end of Chapter I of [16], Grothendieck returns to the same topic and asserts in Corollary 1 (p. 184) that $(\mathrm{G})$ holds even if $X$ itself has the AP. (By a well-known Grothendieck-Enflo-James-Lindenstrauss result (see, e.g., [27, p. 34] the AP of $X^{*}$ implies the AP of $X$, but not vice versa.) This Corollary 1 is an immediate consequence 
of Grothendieck's Theorem 15 (pp. 182-183). However, Grothendieck's proposed proof of Theorem 15 is in error. Indeed, a consequence of his efforts would imply that any Banach space with the AP and the Radon-Nikodým property has the MAP. But [13, Corollary 1.13] exhibits a subspace of $\ell_{1}$ which has the AP but not the BAP.

That the AP of $X$ indeed implies (G), was proven in 2000 by [22], where also the converse was established. This means that the AP is always somewhat metric. The most far-reaching result in this direction can be summarized as follows.

TheOREM 3.1 (cf. [23, 32, 34, 36]). The following assertions are equivalent.

(a) $X$ has the $A P$.

(b) For every Banach space $Y$ and for every operator $T \in \mathcal{W}(Y, X)$, there exists a net $\left(S_{\alpha}\right) \subset \mathcal{F}(X, X)$ such that $\sup _{\alpha}\left\|S_{\alpha} T\right\| \leq\|T\|$ and $T^{*} S_{\alpha}^{*} \rightarrow T^{*}$ in the strong operator topology.

(c) For every separable reflexive Banach space $Z$ and for every operator $T \in \mathcal{K}(Z, X)$, there exists a sequence $\left(T_{n}\right) \subset \mathcal{F}(Z, X)$ such that $T_{n} \rightarrow T$ in the strong operator topology.

In the above-mentioned Corollary 1, Grothendieck also asserted that the AP of $X$ implies the metric approximation of weakly compact operators from $X$ to $Y$, that is, the roles of $X$ and $Y$ can be reversed in $(\mathrm{G})$. This claim is not true: in 1983, O. Reinov [42] constructed a counterexample showing that, in general, even compact operators from $X$ to $Y$ cannot be metrically approximated under the assumption that $X$ has the AP. This paper actually provides a counterexample to the related conjecture of Grothendieck (see [16. Chapter II, p. 135]). However, the metric approximation of weakly compact operators from $X$ to $Y$ is possible whenever $X^{*}$ has the AP (see [34, Theorem 5]).

As was mentioned in Section 2, the AP is not metric in general, but it is not known whether the AP is metric for dual Banach spaces (see Problem 2.1). By a result of Grothendieck [16. Chapter I, Corollary 2 on p. 181], reflexive Banach spaces with the AP enjoy the MAP. By an implicit result of Grothendieck [16. Chapter I, proof of Corollary 2, p. 182, together with Corollary 3, pp. 134-135] (made explicit by [19]) separable dual spaces with the AP also have the MAP. The proof of this result "has always been a little mysterious" as written in [3, p. 289]. The most far-reaching result in this direction is as follows. And even this result can be found implicitly in [16, looking carefully at the above-mentioned erroneous proof, as Reinov did in [41].

Theorem 3.2 (see [9] and [41]). Assume that $X^{*}$ or $X^{* *}$ has the Radon-Nikodym property. If $X^{*}$ has the AP, then $X^{*}$ has the MAP.

Since late 1970s, there have been many different proofs of Theorem 3.2. The proofs in [9], 15], and [41] were modelled after Grothendieck's proofs in [16] (9] considers only the case when $X^{*}$ has the Radon-Nikodým property). For separable dual spaces, an alternative proof is due to Lindenstrauss and Tzafriri [27, pp. 39-40]. The latter proof was adapted in [4] under the assumption that $X^{*}$ has the Radon-Nikodým property. The proofs in [15], 22, and [25] use the description of $\mathcal{K}(X, X)^{*}$ due to Feder and Saphar [11. These proofs "stay" on the level of $X^{*}$. We would like to present a rather unexpected proof from [31] which will "go down" to $X$ and then "up" to $X^{*}$ using Johnson's lifting 
theorem from [17]: if $X$ has the $M A P$ in every equivalent norm, then $X^{*}$ has the MAP. This proof will also use the recent concept of the weak MAP due to [24].

Let $X$ be a Banach space. Following [24, we say that $X$ has the weak $\lambda$-BAP if for every Banach space $Y$ and every operator $T \in \mathcal{W}(X, Y)$, there exists a net $\left(S_{\alpha}\right) \subset$ $\mathcal{F}(X, X)$ such that $S_{\alpha} \rightarrow I_{X}$ uniformly on compact subsets of $X$ and $\lim \sup _{\alpha}\left\|T S_{\alpha}\right\| \leq$ $\lambda\|T\|$. The weak MAP is the weak 1-BAP. The weak BAP is the weak $\lambda$-BAP for some $\lambda$.

The $\lambda$-BAP clearly implies the weak $\lambda$-BAP, which in turn implies the AP (take $T=0$ in the above). Using the description of $\mathcal{K}(X, X)^{*}$ from [11, the following can be proven. THEOREM 3.3 (31]). If $X^{*}$ or $X^{* *}$ has the Radon-Nikodym property, then the weak $\lambda-B A P$ and the $\lambda-B A P$ are equivalent for $X$.

It remains open whether the weak $\lambda$-BAP is strictly weaker than the $\lambda$-BAP. If they were equivalent, then the answer to Problem 2.1 would be "yes" by the following result which shows that the AP of a dual space is always weakly metric.

TheOREm 3.4 ([24]). The AP and the weak MAP are equivalent for $X^{*}$.

Another "metric" characterization of the AP of a dual space is as follows.

THEOREM 3.5 ([24]). The dual space $X^{*}$ has the AP if and only if $X$ has the weak MAP in all its equivalent renormings.

The promised proof of Theorem 3.2 goes like this. Since $X^{*}$ has the AP, $X$ has the weak MAP in all its equivalent renormings (by Theorem 3.5). Since the Radon-Nikodým property is invariant under isomorphisms, by Theorem 3.3, $X$ has the MAP in all its equivalent renormings. But in this case, $X^{*}$ already has the MAP by Johnson's lifting theorem.

Before discussing the essence of the weak BAP in the subsequent Sections 4 and 5 , let us describe the main tool which has been used in the proofs of Theorems 3.1, 3.3-3.5, and of the related results from [22. This tool is an isometric version of the famous DavisFigiel-Johnson-Pełczyński factorization lemma [5] due to Å. Lima, O. Nygaard, and Oja 22 ; see the Factorization Lemma below. In the above-mentioned proofs, the Factorization Lemma has been applied in rather different situations and, in summa summarum, probably all aspects of it have been needed.

Let $\mathbf{a}$ be the unique solution of the equation

$$
\sum_{n=1}^{\infty} \frac{a^{n}}{\left(a^{n}+1\right)^{2}}=1, a>1 .
$$

Let $X$ be a Banach space and let $K$ be a closed absolutely convex subset of $B_{X}$, the closed unit ball of $X$. For each $n \in N$, put $B_{n}=\mathbf{a}^{n / 2} K+\mathbf{a}^{-n / 2} B_{X}$. The gauge of $B_{n}$ gives an equivalent norm $\|\cdot\|_{n}$ on $X$. Set

$$
\|x\|_{K}=\left(\sum_{n=1}^{\infty}\|x\|_{n}^{2}\right)^{1 / 2},
$$

define $X_{K}=\left\{x \in X:\|x\|_{K}<\infty\right\}$, and let $J_{K}: X_{K} \rightarrow X$ denote the identity embedding. FACTORIZATION LEMma 3.6 (see [5] and [22]). With notation as above, the following holds. 
(i) $X_{K}=\left(X_{K},\|\cdot\|_{K}\right)$ is a Banach space and $\left\|J_{K}\right\| \leq 1$.

(ii) $K \subset B_{X_{K}} \subset B_{X}$.

(iii) For $x \in K$, one has $\|x\|_{K}^{2} \leq(1 / 4+1 / \ln \mathbf{a})\|x\|$.

(iv) $B_{X_{K}} \subset B_{n}$ for all $n \in N$.

(v) $J_{K}^{*}\left(X^{*}\right)$ is norm dense in $X_{K}^{*}$.

(vi) $J_{K}$ is compact if and only if $K$ is compact; then $X_{K}$ is separable.

(vii) $X_{K}$ is reflexive if and only if $K$ is weakly compact.

4. The weak BAP. Let us fix some more notation. We denote by $\mathcal{I}$ and $\mathcal{N}$ the operator ideals of integral and nuclear operators. The projective tensor norm will be denoted by $\pi$. We shall occasionally need also the following operator ideals: $\mathcal{N}_{p}-p$-nuclear operators, $\mathcal{I}_{p}-p$-integral operators, $\mathcal{P}_{p}-$ absolutely $p$-summing operators ( $p$-summing in [8])(see [40] or 43]). We refer to Ryan's book [43] for tensor norms and to the books by DiestelJarchow-Tonge [8] and Pietsch [40] for operator ideals. Recall that a Banach operator ideal $\mathcal{A}$ is regular if $T \in \mathcal{A}(X, Y)$ and $\|T\|_{\mathcal{A}(X, Y)}=\|T\|_{\mathcal{A}\left(X, Y^{* *}\right)}$ whenever $T \in \mathcal{L}(X, Y)$ and $T \in \mathcal{A}\left(X, Y^{* *}\right)$. We denote by $\mathcal{A}^{*}$ the dual operator ideal of $\mathcal{A}$. Its components are $\mathcal{A}^{*}(X, Y)=\left\{T \in \mathcal{L}(X, Y): T^{*} \in \mathcal{A}\left(Y^{*}, X^{*}\right)\right\}$ with $\|T\|_{\mathcal{A}^{*}}=\left\|T^{*}\right\|_{\mathcal{A}}$. (The notation $\mathcal{A}^{*}$ is reserved for another concept in [40] where the dual operator ideal is denoted by $\mathcal{A}^{\text {dual }}$.)

The BAP of a Banach space $X$ is defined in terms of the space $X$ (see Section 2). The definition of the weak BAP of $X$ uses all weakly compact operators from $X$ to arbitrary Banach spaces $Y$ (see Section 3). In a recent work [33], the weak BAP of $X$ is also described in terms of the space $X$ itself without having recourse to other Banach spaces $Y$. Let us see how this is done.

Our starting point is formed by two theorems which are essentially due to Grothendieck [16, Chapter I, p. 179] (see, e.g., [43, p. 80]) and to Lima and Oja [24]. We state them in an equivalent form using inequalities on finite-rank operators.

THEOREM 4.1 (Grothendieck). The following assertions are equivalent.

(a) $X$ has the $\lambda$-BAP.

(b) $\|S\|_{\pi} \leq \lambda\|S\|_{\mathcal{I}(X, X)}$ for all $S \in \mathcal{F}(X, X)$.

(c) $\|T\|_{\pi} \leq \lambda\|T\|_{\mathcal{I}(Y, X)}$ for all Banach spaces $Y$ and for all $T \in \mathcal{F}(Y, X)$.

TheOREM 4.2 (cf. [24, Theorem 3.2]). The following assertions are equivalent.

(a) $X$ has the weak $\lambda$-BAP.

(b) $\|T\|_{\pi} \leq \lambda\|T\|_{\mathcal{I}(Z, X)}$ for all reflexive Banach spaces $Z$ and for all $T \in \mathcal{F}(Z, X)$.

One may say that Theorem 4.1 characterizes the BAP both through an "inner" and an "outer" inequalities, but Theorem 4.2 characterizes the weak BAP through a restricted "outer" inequality. We would like to characterize the weak BAP also through an "inner" inequality. To make it precise, let us introduce the following terminology (see [33]).

If $X$ is a closed subspace of a Banach space $W, \alpha$ is a tensor norm, $\mathcal{A}$ is a Banach operator ideal, and $\lambda>0$, then we shall call the condition

$$
\|S\|_{\alpha} \leq \lambda\|S\|_{\mathcal{A}(X, W)} \quad \text { for all } \quad S \in \mathcal{F}(X, X)
$$


an inner inequality, and the condition

$$
\|T\|_{\alpha} \leq \lambda\|T\|_{\mathcal{A}(Y, W)} \quad \text { for all Banach spaces } Y \text { and for all } T \in \mathcal{F}(Y, X)
$$

an outer inequality.

Now, how to pass from an inner inequality $\|\cdot\|_{\alpha} \leq\|\cdot\|_{\mathcal{A}}$ to the corresponding outer inequality? Our first result shows that the inner inequality $\|\cdot\|_{\pi} \leq \lambda\|\cdot\|_{\mathcal{A}}$ always implies a restricted outer inequality (with respect to reflexive spaces) and, under some natural restrictions on $\mathcal{A}$, it implies the outer inequality. Below, $X$ will be a closed subspace of a Banach space $W$.

Proposition $4.3([33)$. Let $\mathcal{A}$ be a Banach operator ideal. If

$$
\|S\|_{\pi} \leq \lambda\|S\|_{\mathcal{A}(X, W)}
$$

for all $S \in \mathcal{F}(X, X)$, then

$$
\|T\|_{\pi} \leq \lambda\|T\|_{\mathcal{A}(Z, W)}
$$

for all reflexive Banach spaces $Z$ and for all $T \in \mathcal{F}(Z, X)$. If, moreover, $\mathcal{A}$ is regular and satisfies $\mathcal{A} \subset \mathcal{A}^{* *}$, then

$$
\|T\|_{\pi} \leq \lambda\|T\|_{\mathcal{A}(Y, W)}
$$

for all Banach spaces $Y$ and for all $T \in \mathcal{F}(Y, X)$.

The "moreover" part applies to many important operator ideals, such as $\mathcal{I}=\mathcal{I}_{1}$, $\mathcal{I}_{p}$ and $\mathcal{P}_{p}, 1 \leq p \leq \infty$. In particular, taking $W=X$ and $\mathcal{A}=\mathcal{I}$ yields the implication (b) $\Rightarrow$ (c) of Grothendieck's Theorem 4.1. This might be considered as an alternative direct proof of it, since the proofs in the literature pass through condition (a) of Theorem 4.1 (see, e.g., [6, 16.3], [9, pp. 243-244], or [43, pp. 80-81]) as the original proof in [16, Chapter I, pp. 179-180] does.

However, the "moreover" part does not apply to $\mathcal{N}_{p}$. Our next result (its proof relies on the Factorization Lemma) shows that, in this case, the outer inequality $\|\cdot\|_{\alpha} \leq \lambda\|\cdot\|_{\mathcal{N}_{p}}$ is fully determined by the class of separable reflexive Banach spaces for any tensor norm $\alpha$.

TheOREM 4.4 ([33]). Let $\alpha$ be a tensor norm and let $1 \leq p \leq \infty$. If

$$
\|S\|_{\alpha} \leq \lambda\|S\|_{\mathcal{N}_{p}(Z, W)}
$$

for all separable reflexive Banach spaces $Z$ and for all $S \in \mathcal{F}(Z, X)$, then

$$
\|T\|_{\alpha} \leq \lambda\|T\|_{\mathcal{N}_{p}(Y, W)}
$$

for all Banach spaces $Y$ and for all $T \in \mathcal{F}(Y, X)$.

Applying first Proposition 4.3 and then Theorem 4.4 yields the following result.

COROLlaRY 4.5 ([33]). The following assertions are equivalent.

(a) $\|S\|_{\pi} \leq \lambda\|S\|_{\mathcal{N}(X, W)}$ for all $S \in \mathcal{F}(X, X)$.

(b) $\|T\|_{\pi} \leq \lambda\|T\|_{\mathcal{N}(Z, W)}$ for all separable reflexive Banach spaces $Z$ and for all $T \in$ $\mathcal{F}(Z, X)$.

(c) $\|T\|_{\pi} \leq \lambda\|T\|_{\mathcal{N}(Y, W)}$ for all Banach spaces $Y$ and for all $T \in \mathcal{F}(Y, X)$. 
Let us consider two special cases $W=X$ and $W=X^{* *}$ of Corollary 4.5. The first case brings us to the AP, giving, in particular, an alternative proof (cf., e.g., [43, p. 76, Corollary 4.8]) for Grothendieck's characterizations of the AP for $X$ such as $\|S\|_{\pi}=\|S\|_{\mathcal{N}(X, X)}$ for all $S \in \mathcal{F}(X, X)$, and $\|T\|_{\pi}=\|T\|_{\mathcal{N}(Y, X)}$ for all Banach spaces $Y$ and for all $T \in$ $\mathcal{F}(Y, X)$. Using Theorem 4.2 (and the fact that $\|T\|_{\mathcal{N}\left(Z, X^{* *}\right)}=\|T\|_{\mathcal{I}\left(Z, X^{* *}\right)}=\|T\|_{\mathcal{I}(Z, X)}$ if $Z$ is reflexive and $T \in \mathcal{F}(Z, X))$, the case $W=X^{* *}$ yields new characterizations of the weak BAP below.

THEOREM 4.6 ([33]). The following assertions are equivalent.

(a) $X$ has the weak $\lambda$-BAP.

(b) $\|S\|_{\pi} \leq \lambda\|S\|_{\mathcal{N}\left(X, X^{* *}\right)}$ for all $S \in \mathcal{F}(X, X)$.

(c) $\|T\|_{\pi} \leq \lambda\|T\|_{\mathcal{N}\left(Y, X^{* *}\right)}$ for all Banach spaces $Y$ and for all $T \in \mathcal{F}(Y, X)$.

Condition (b) of Theorem 4.6 appears to be the essence of the weak BAP: it opens the way to remarkably simple proofs of many basic results concerning the weak BAP. For instance, Theorem 3.3 holds, by Theorem 4.6 (b) and Theorem 4.1 (b), because all $S \in \mathcal{F}(X, X)$ satisfy

$$
\|S\|_{\pi} \leq \lambda\|S\|_{\mathcal{N}\left(X, X^{* *}\right)}=\lambda\|S\|_{\mathcal{I}\left(X, X^{* *}\right)}=\lambda\|S\|_{\mathcal{I}(X, X)}
$$

(the first equality holds since $X^{*}$ or $X^{* *}$ has the Radon-Nikodým property). Theorem 3.4 holds, by Theorem 4.6 (b), because all $S \in \mathcal{F}\left(X^{*}, X^{*}\right)$ satisfy

$$
\|S\|_{\pi}=\|S\|_{\mathcal{N}\left(X^{*}, X^{*}\right)}=\|S\|_{\mathcal{N}\left(X^{*}, X^{* * *}\right)}
$$

(the first equality holds since $X^{*}$ has the AP, the second one holds since $X^{*}$ is 1complemented in $\left.X^{* * *}\right)$. Concerning Theorem 3.5, the AP of $X^{*}$ implies the weak MAP of $X$ (this implication was used in the proof of Theorem 3.2 in Section 3), by Theorem 4.6 (b), because all $S \in \mathcal{F}(X, X)$ satisfy

$$
\|S\|_{\pi}=\|S\|_{X^{*} \otimes_{\pi} X}=\|S\|_{X^{*} \otimes_{\pi} X^{* *}}=\left\|S^{*}\right\|_{\pi}=\left\|S^{*}\right\|_{\mathcal{N}\left(X^{*}, X^{*}\right)}=\|S\|_{\mathcal{N}\left(X, X^{* *}\right)} .
$$

5. The AP which is bounded for a Banach operator ideal. Recall that $\mathcal{L}, \mathcal{W}$, and $\mathcal{K}$ denote the Banach operator ideals of bounded, weakly compact, and compact linear operators, respectively.

The weak BAP is defined (see Section 3) as an AP which is bounded for every weakly compact operator. In other words, the weak BAP is an AP which is bounded for $\mathcal{W}$. This suggests a general definition that might be used to unify various ideas related to different variants of APs and to connections between them.

Let $X$ be a Banach space and let $\mathcal{A}=\left(\mathcal{A},\|\cdot\|_{\mathcal{A}}\right)$ be a Banach operator ideal. Following 21], we say that $X$ has the $\lambda$-bounded approximation property for $\mathcal{A}(\lambda$-BAP for $\mathcal{A})$ if for every Banach space $Y$ and every operator $T \in \mathcal{A}(X, Y)$, there exists a net $\left(S_{\alpha}\right) \subset \mathcal{F}(X, X)$ such that $S_{\alpha} \rightarrow I_{X}$ uniformly on compact subsets of $X$ and

$$
\limsup _{\alpha}\left\|T S_{\alpha}\right\|_{\mathcal{A}} \leq \lambda\|T\|_{\mathcal{A}} .
$$

The notion of the BAP for $\mathcal{A}$ seems to be the first attempt to define an AP related to a Banach operator ideal $\mathcal{A}$ that also depends on the operator ideal norm of $\mathcal{A}$, and not only on the operator ideal properties of $\mathcal{A}$. Recall that there are well-known variations 
of the BAP involving operator ideals which have been studied since the early 1980s (see, e.g., 28 for references). Let $\mathcal{A}$ be an operator ideal. A Banach space $X$ is said to have the $\lambda$-bounded $\mathcal{A}$-approximation property $(\lambda$-bounded $\mathcal{A}$-AP) if there exists a net $\left(S_{\alpha}\right) \subset \mathcal{A}(X, X)$ with $\sup _{\alpha}\left\|S_{\alpha}\right\| \leq \lambda$ such that $S_{\alpha} \rightarrow I_{X}$ uniformly on compact subsets of $X$.

The bounded $\mathcal{K}$-AP is called the bounded compact approximation property (BCAP). This well-known notion goes back to Banach's book where the question whether all separable Banach spaces have the BCAP was essentially posed (see [2, p. 237]). If $X$ has the AP and the BCAP, then $X$ already has the BAP (see, e.g., [3, Proposition 8.2]). Therefore, the subspace of $c_{0}$, constructed in [13] (see Section 2), which fails the BAP but has the AP, also fails the BCAP. The Banach space $X_{W}$, constructed by G. Willis [44, has the MCAP (in fact, it has the commuting MCAP (see [35])) but fails the AP. Therefore $X_{W}$ fails the BAP for any Banach operator ideal $\mathcal{A}$.

It would be very restrictive to define the notion of the $\lambda$-bounded $\mathcal{A}$-AP by using the operator ideal norm $\|\cdot\|_{\mathcal{A}}$ instead of the usual operator norm $\|\cdot\|$, as was noted in [30]. For instance, even $\ell_{2}$ would not have the bounded $\mathcal{N}$-AP. Indeed, assume that there would exist a net $\left(S_{\alpha}\right) \subset \mathcal{N}\left(\ell_{2}, \ell_{2}\right)$ with $\sup _{\alpha}\left\|S_{\alpha}\right\|_{\mathcal{N}} \leq \lambda$ such that $S_{\alpha} \rightarrow I_{\ell_{2}}$ uniformly on compact subsets of $\ell_{2}$. Denote by $i_{n}: \ell_{2}^{n} \rightarrow \ell_{2}$ the natural embedding and by $p_{n}: \ell_{2} \rightarrow \ell_{2}^{n}$ the natural projection. Then, for the identity operator $I_{n}$ on $\ell_{2}^{n}$, we would have that $p_{n} S_{\alpha} i_{n} \rightarrow I_{n}$ uniformly on compact subsets of $\ell_{2}^{n}$. Since $\ell_{2}^{n}$ is of finite dimension,

$$
\left\|I_{n}\right\|_{\mathcal{N}}=\lim _{\alpha}\left\|p_{n} S_{\alpha} i_{n}\right\|_{\mathcal{N}} \leq \sup _{\alpha}\left\|p_{n}\right\|\left\|S_{\alpha}\right\|_{\mathcal{N}}\left\|i_{n}\right\|=\sup _{\alpha}\left\|S_{\alpha}\right\|_{\mathcal{N}} \leq \lambda,
$$

which is impossible for $n>\lambda$ because $\left\|I_{n}\right\|_{\mathcal{N}}=n$.

The weak $\lambda$-BAP is, by definition, the $\lambda$-BAP for $\mathcal{W}$. In [24], it is proven that the weak $\lambda$-BAP is also the same as the $\lambda$-BAP for $\mathcal{K}$. The $\lambda$-BAP clearly implies the $\lambda$-BAP for every Banach operator ideal $\mathcal{A}$ (since $\left\|T S_{\alpha}\right\|_{\mathcal{A}} \leq\|T\|_{\mathcal{A}}\left\|S_{\alpha}\right\| \leq \lambda\|T\|_{\mathcal{A}}$ whenever $\left.\left\|S_{\alpha}\right\| \leq \lambda\right)$, in particular, $\ell_{2}$ has the 1 -BAP for every $\mathcal{A}$. Since $I_{X} \in \mathcal{L}(X, X)$, the BAP is the same as the BAP for $\mathcal{L}$. In general, $I_{X} \notin \mathcal{A}(X, X)$. For instance, $I_{X} \in \mathcal{W}(X, X)$ means that $X$ is reflexive, $I_{X} \in \mathcal{K}(X, X)$ or $I_{X} \in \mathcal{I}(X, X)$ means that $\operatorname{dim} X<\infty$.

In 21], the following reformulations of the BAP in terms of the boundedness for the Banach operator ideals $\mathcal{S I}$ and $\mathcal{I}$ of strictly integral and integral operators were established. (Following [8, we use the term "strictly integral operator" for "Pietsch integral operator" in [9] and [43].)

THEOREM 5.1 (21]). A Banach space $X$ has the $\lambda-B A P$ if and only if $X$ has the $\lambda-B A P$ for $\mathcal{S I}$ if and only if $X$ has the $\lambda-B A P$ for $\mathcal{I}$.

For the weak BAP, the following holds.

TheOrem 5.2 ([21]). A Banach space $X$ has the weak $\lambda$-BAP if and only if $X$ has the $\lambda-B A P$ for $\mathcal{N}$.

Sketch of the proof of Theorem 5.1 (cf. [21, proof of Theorem 2.1]). First of all, recall that the $\lambda$-BAP implies the $\lambda$-BAP for every $\mathcal{A}$. Now assume that $X$ has the $\lambda$-BAP for $\mathcal{I}$ or $\mathcal{S I}$. Then, by the definition (we use it for $\left.Y=\ell_{1}(\Gamma)^{* *}\right)$, for every $T \in \mathcal{I}\left(X, \ell_{1}(\Gamma)^{* *}\right)=$ 
$\mathcal{S I}\left(X, \ell_{1}(\Gamma)^{* *}\right)$, there exists a net $\left(S_{\alpha}\right) \subset \mathcal{F}(X, X)$ such that $S_{\alpha} \rightarrow I_{X}$ pointwise and $\limsup \sup _{\alpha}\left\|T S_{\alpha}\right\|_{\mathcal{I}} \leq \lambda\|T\|_{\mathcal{I}}$. Since $T S_{\alpha}$ is a finite-rank operator whose range space is a dual space having the MAP, we have $\left\|T S_{\alpha}\right\|_{\mathcal{I}}=\left\|T S_{\alpha}\right\|_{\pi}$. But this yields a property which passes from $\ell_{1}(\Gamma)$ to quotient spaces of $\ell_{1}(\Gamma)$ (see [21, Lemma 2.3]). Therefore, using that $X$ is a quotient of some $\ell_{1}(\Gamma)$-space, we may assume that for every $U \in \mathcal{I}\left(X, X^{* *}\right)$ there exists a net $\left(S_{\alpha}\right) \subset \mathcal{F}(X, X)$ such that $S_{\alpha} \rightarrow I_{X}$ pointwise and

$$
\lim \sup _{\alpha}\left\|U S_{\alpha}\right\|_{\pi} \leq \lambda\|U\|_{\mathcal{I}}
$$

To choose the operator $U$, let us recall that we need to show that $X$ has the $\lambda$-BAP. By Theorem 4.1, this means that $\|S\|_{\pi} \leq \lambda\|S\|_{\mathcal{I}}$ for all $S \in \mathcal{F}(X, X)$. So let $S \in \mathcal{F}(X, X)$. By [21, Lemma 2.4], there exist $A \in \mathcal{L}\left(X^{*}, X^{*}\right)$ with $\|A\|=1$ and $V \in \mathcal{F}(X, X)$ such that $V^{*}=A S^{*}$ and

$$
\|S\|_{\pi} \leq \limsup _{\alpha}\left\|j_{X} V S_{\alpha}\right\|_{\pi}
$$

where $j_{X}: X \rightarrow X^{* *}$ denotes the canonical embedding and $\left(S_{\alpha}\right) \subset \mathcal{F}(X, X)$ is any net converging pointwise to the identity $I_{X}$. Take $U=j_{X} V$ and find $\left(S_{\alpha}\right)$ as above. Then

$$
\|S\|_{\pi} \leq \lambda\left\|j_{X} V\right\|_{\mathcal{I}}=\lambda\left\|V^{*}\right\|_{\mathcal{I}}=\lambda\left\|A S^{*}\right\|_{\mathcal{I}} \leq \lambda\|A\|\left\|S^{*}\right\|_{\mathcal{I}}=\lambda\|S\|_{\mathcal{I}}
$$

as desired.

Concerning the proof of Theorem 5.2 (cf. [21, proof of Theorem 3.1]), to show that $X$ has the weak $\lambda$-BAP, one relies on its characterization (b) in Theorem 4.6. For the converse implication, one proceeds from another recent criterion of the weak BAP, which is formulated in terms of extension operators from $X^{*}$ to $X^{* * *}$ (see [26, Propositions 2.1, 2.3, and 2.5] and [33, Corollary 3.18]).

There is a natural partial ordering on the class of all Banach operator ideals: for Banach operator ideals $\mathcal{A}$ and $\mathcal{B}$, the inclusion $\mathcal{A} \subset \mathcal{B}$ means that $\mathcal{A}(X, Y) \subset \mathcal{B}(X, Y)$ and $\|T\|_{\mathcal{A}} \geq\|T\|_{\mathcal{B}}$ for all Banach spaces $X$ and $Y$, and for all operators $T \in \mathcal{A}(X, Y)$. Here $\mathcal{N}$ is the smallest element and $\mathcal{L}$ is the largest element.

Every Banach operator ideal $\mathcal{A}$ yields some kind of the AP, namely the BAP for $\mathcal{A}$. Let us look at the chain

$$
\mathcal{N} \subset \mathcal{S I} \subset \mathcal{I} \subset \mathcal{W} \subset \mathcal{L}
$$

From the above, we know that $\mathcal{N}$ yields the weak BAP, but its continuous analogue $\mathcal{S} \mathcal{I}$ yields the BAP, as does $\mathcal{I}$. Then $\mathcal{W}$ yields again the weak BAP. And, in turn, $\mathcal{L}$ yields the BAP.

With the ample choice of different Banach operator ideals, many natural questions arise. Let us look at some of them.

"Zooming in" between $\mathcal{I}$ and $\mathcal{W}$, one can see, e.g., the Banach operator ideal $\mathcal{P}$ of absolutely summing operators and its dual ideal $\mathcal{P}^{*}$. In [21], it is shown that $\mathcal{P}^{*}$ yields the BAP.

\section{Problem 5.3. Describe the BAP for $\mathcal{P}$.}

More generally, recalling that $\mathcal{N}=\mathcal{N}_{1}, \mathcal{I}=\mathcal{I}_{1}, \mathcal{P}=\mathcal{P}_{1}$, one is interested in the following.

Problem 5.4. Describe the BAP for $\mathcal{N}_{p}, \mathcal{I}_{p}, \mathcal{P}_{p}, 1<p \leq \infty$. 
A Banach operator ideal is called classical (in the terminology of [8]) or closed (in the terminology of [40]) if its operator ideal norm is the usual operator norm. Classical Banach operator ideals are, e.g., $\mathcal{K}, \mathcal{W}, \mathcal{L}$, the ideal $\mathcal{R} \mathcal{N}$ of Radon-Nikodým operators (see, e.g., [40, p. 337]), the ideal $\mathcal{U}$ of unconditionally summing operators, the ideal $\mathcal{V}$ of completely continuous operators. As was mentioned, $\mathcal{K}$ and $\mathcal{W}$ yield the weak BAP and $\mathcal{L}$ yields the BAP. Here we have the chains $\mathcal{K} \subset \mathcal{W} \subset \mathcal{R N} \subset \mathcal{U} \subset \mathcal{L}$ and $\mathcal{K} \subset \mathcal{V} \subset \mathcal{U}$

Problem 5.5. Describe the BAP for $\mathcal{R} \mathcal{N}, \mathcal{V}, \mathcal{U}$.

We do not know what is happening between $\mathcal{W}$ and $\mathcal{L}$. In particular, one may ask as follows.

Problem 5.6 ([21]). Is there the largest of the classical Banach operator ideals yielding the weak BAP? Is there the smallest of the classical Banach operator ideals yielding the $B A P$ ?

Acknowledgements. The author thanks the referee for helpful suggestions that improved the exposition. This paper is based on a lecture delivered at the $19^{\text {th }}$ International Conference on Banach Algebras held at Będlewo, July 14-24, 2009. The support for the meeting by the Polish Academy of Sciences, the European Science Foundation under the ESF-EMS-ERCOM partnership, and the Faculty of Mathematics and Computer Science of the Adam Mickiewicz University at Poznań is gratefully acknowledged. The research was partially supported by Estonian Science Foundation Grant 7308 and Estonian Targeted Financing Project SF0180039s08.

\section{References}

[1] G. Alberti, M. Csörnyei, A. Pełczyński, and D. Preiss, BV has the bounded approximation property, J. Geom. Anal. 15 (2005), 1-7.

[2] S. Banach, Théorie des opérations linéaires, Monografie Matematyczne, Warszawa, 1932.

[3] P. G. Casazza, Approximation properties, in: W. B. Johnson and J. Lindenstrauss (eds.), Handbook of the Geometry of Banach Spaces, Vol. 1, Elsevier, 2001, 271-316.

[4] Ch.-M. Cho and W. B. Johnson, A characterization of subspaces $X$ of $\ell_{p}$ for which $K(X)$ is an $M$-ideal in $L(X)$, Proc. Amer. Math. Soc. 93 (1985), 466-470.

[5] W. J. Davis, T. Figiel, W. B. Johnson, and A. Pełczyński, Factoring weakly compact operators, J. Funct. Anal. 17 (1974), 311-327.

[6] A. Defant and K. Floret, Tensor Norms and Operator Ideals, North-Holland Mathematics Studies 176 (1993).

[7] J. Diestel, J. H. Fourie, and J. Swart, The Metric Theory of Tensor Products. Grothendieck's Résumé Revisited, Amer. Math. Soc., Providence, RI, 2008.

[8] J. Diestel, H. Jarchow, and A. Tonge, Absolutely Summing Operators, Cambridge University Press, Cambridge, 1995.

[9] J. Diestel and J. J. Uhl, Jr. Vector Measures, Mathematical Surveys 15, Amer. Math. Soc., Providence, RI, 1977.

[10] P. Enflo, A counterexample to the approximation problem in Banach spaces, Acta Math. 130 (1973), 309-317. 
[11] M. Feder and P. D. Saphar, Spaces of compact operators and their dual spaces, Israel J. Math. 21 (1975), 38-49.

[12] T. Figiel and W. B. Johnson, The approximation property does not imply the bounded approximation property, Proc. Amer. Math. Soc. 41 (1973), 197-200.

[13] T. Figiel, W. B. Johnson, and A. Pełczyński, Some approximation properties of Banach spaces and Banach lattices, Israel J. Math. (to appear).

[14] G. Godefroy, The Banach space c $c_{0}$, Extracta Math. 16 (2001), 1-25.

[15] G. Godefroy and P. D. Saphar, Duality in spaces of operators and smooth norms on Banach spaces, Illinois J. Math. 32 (1988), 672-695.

[16] A. Grothendieck, Produits tensoriels topologiques et espaces nucléaires, Mem. Amer. Math. Soc. 16 (1955).

[17] W. B. Johnson, A complementary universal conjugate Banach space and its relation to the approximation problem, Israel J. Math. 13 (1972), 301-310.

[18] W. B. Johnson and T. Oikhberg, Separable lifting property and extensions of local reflexivity, Illinois J. Math. 45 (2001), 123-137.

[19] W. B. Johnson, H. P. Rosenthal, and M. Zippin, On bases, finite dimensional decompositions and weaker structures in Banach spaces, Israel J. Math. 9 (1971), 488-506.

[20] R. Kałuża, Through a Reporter's Eyes: the Life of Stefan Banach, Birkhäuser, Boston, 1996.

[21] Å. Lima, V. Lima, and E. Oja, Bounded approximation properties via integral and nuclear operators, Proc. Amer. Math. Soc. 138 (2010), 287-297.

[22] A. Lima, O. Nygaard, and E. Oja, Isometric factorization of weakly compact operators and the approximation property, Israel J. Math. 119 (2000), 325-348.

[23] A. Lima and E. Oja, Ideals of operators, approximability in the strong operator topology, and the approximation property, Michigan Math. J. 52 (2004), 253-265.

[24] A. Lima and E. Oja, The weak metric approximation property, Math. Ann. 333 (2005), 471-484.

[25] Å. Lima and E. Oja, Metric approximation properties and trace mappings, Math. Nachr. 280 (2007), 571-580.

[26] V. Lima, The weak metric approximation property and ideals of operators, J. Math. Anal. Appl. 334 (2007), 593-603.

[27] J. Lindenstrauss and L. Tzafriri, Classical Banach Spaces I, Ergebnisse der Mathematik und ihrer Grenzgebiete 92, Springer-Verlag, Berlin-Heidelberg-New York, 1977.

[28] A. Lissitsin, K. Mikkor, and E. Oja, Approximation properties defined by spaces of operators and approximability in operator topologies, Illinois J. Math. 52 (2008), 563-582.

[29] R. D. Mauldin (ed.), The Scottish Book: Mathematics from the Scottish Café, Birkhäuser, Boston, 1981.

[30] E. Oja, Lifting bounded approximation properties from Banach spaces to their dual spaces, J. Math. Anal. Appl. 323 (2006), 666-679.

[31] E. Oja, The impact of the Radon-Nikodym property on the weak bounded approximation property, Rev. R. Acad. Cien. Serie A. Mat. 100 (2006), 325-331.

[32] E. Oja, The strong approximation property, J. Math. Anal. Appl. 338 (2008), 407-415.

[33] E. Oja, Inner and outer inequalities with applications to approximation properties, Trans. Amer. Math. Soc. (to appear).

[34] E. Oja and A. Pelander, The approximation property in terms of the approximability of weak*-weak continuous operators, J. Math. Anal. Appl. 286 (2003), 713-723.

[35] E. Oja and I. Zolk, On commuting approximation properties of Banach spaces, Proc. Royal Soc. Edinburgh 139A (2009), 551-565.

[36] A. Pelander, The approximation property in terms of density in operator topologies, Acta Comment. Univ. Tartuensis Math. 7 (2003), 37-44. 
[37] A. Pełczyński, On some problems of Banach, Uspekhi Mat. Nauk 28 (1973), 67-75 (in Russian).

[38] A. Pełczyński and T. Figiel, On Enflo's method of construction of Banach spaces without the approximation property, Uspekhi Mat. Nauk 28 (1973), 95-108 (in Russian).

[39] A. Pełczyński and M. Wojciechowski, Spaces of functions with bounded variation and Sobolev spaces without local unconditional structure, J. Reine Angew. Math. 558 (2003), 109-159.

[40] A. Pietsch, Operator Ideals, North-Holland Publishing Company, Amsterdam-New YorkOxford, 1980.

[41] O. I. Reinov, Operators of RN type in Banach spaces, Sibirsk. Mat. Zh. 19 (1978), 857-865 (in Russian).

[42] O. I. Reinov, Un contre-exemple à une conjecture de A. Grothendieck, C. R. Acad. Sc. Paris, Sér. I, 296 (1983), 597-599.

[43] R. A. Ryan, Introduction to Tensor Products of Banach Spaces, Springer Monographs in Mathematics, Springer-Verlag, London, 2002.

[44] G. Willis, The compact approximation property does not imply the approximation property, Studia Math. 103 (1992), 99-108.

[45] I. Zolk, The Johnson-Schechtman space has the 6-bounded approximation property, J. Math. Anal. Appl. 358 (2009), 493-495. 
